\title{
The Rate at which Energy Decays in a String Damped at One End
}

\author{
Steven Cox 8 EnRique Zuazua
}

\begin{abstract}
The energy in a nonhomogeneous string that moves freely at one end and against unit friction at the other is known to decay exponentially in time. The energy in fact vanishes in finite time when the string's density is uniformly one. We show that this is the only distribution of mass for which the energy vanishes in finite time. Assuming that the density is not one at the damped end and that it has two square integrable derivatives we identify the best rate of decay with the supremum of the real part of the spectrum of the infinitesimal generator of the underlying semigroup. Careful estimation of this spectrum permits us to establish the existence of a density that minimizes the decay rate over a large class of competitors.
\end{abstract}

\section{Introduction}

The displacement $u$ of a string of unit length and density $\rho^{2}$, free at the left end, and in the presence of viscous damping at the right end, satisfies

$$
\begin{aligned}
\rho^{2}(x) u_{t t}(x, t)-u_{x x}(x, t) & =0, \quad 0<x<1,0<t, \\
u_{x}(0, t)=u_{x}(1, t)+u_{t}(1, t) & =0, \quad 0<t,
\end{aligned}
$$

upon being set in motion by the initial disturbance

$$
u(x, 0)=u_{0}(x), \quad u_{t}(x, 0)=v_{0}(x),
$$

assumed an element of the energy space $X=H^{1}(0,1) \times L^{2}(0,1)$ with inner product

$$
\langle[u, v],[w, z]\rangle=\int_{0}^{1} u^{\prime} \bar{w}^{\prime}+u \bar{w}+v \bar{z} d x .
$$


We assume throughout that $\rho$ is measurable and that

$$
0<\alpha \leq \rho(x) \leq \beta<\infty \quad \text { a.e. in } \quad(0,1) .
$$

Under the additional assumption that $\rho$ is of bounded variation, the energy in the string at time $t$,

$$
E(t)=\int_{0}^{1} u_{x}^{2}(x, t)+\rho^{2}(x) u_{t}^{2}(x, t) d x
$$

is known to obey $E(t) \leq C E(0) e^{2 \omega t}$ for some finite $C>0$ and $\omega<0$, independent of the chosen initial data. We establish this in the appendix via "sideways" energy estimates, a technique that goes back, at least, to the work of Rauch and Taylor [19] (see also Symes [21] and Zuazua [24]).

Let us observe, however, that the quantity

$$
f(t)=u(1, t)+\int_{0}^{1} \rho^{2} u_{t} d x
$$

remains constant along the trajectories. This is due to the lack of coercivity of the energy $E$ in the space $X$. Given any initial data $\left[u_{0}, u_{1}\right]$ we may decompose it as $\left[u_{0}, u_{1}\right]=\left[\tilde{u}_{0}, u_{1}\right]+[f(0), 0]$ where $\tilde{u}_{0}=u_{0}-f(0)$. Then the solution $\left[u, u_{t}\right]$ of $(1.1)$ can be written $\left[u, u_{t}\right]=\left[\tilde{u}, u_{t}\right]+[f(0), 0]$ where $\left[\tilde{u}, u_{t}\right]$ is the solution of (1.1) with initial data $\left[\tilde{u}_{0}, u_{1}\right]$ for which the corresponding quantity $f$ vanishes, i.e., $f(t)=0$ for all $t \geq 0$.

The fact that the energy decays exponentially to zero is equivalent to the exponential convergence, in $X$ as $t \rightarrow \infty$, of the trajectory $\left[u, u_{t}\right]$ to the equilibrium configuration $[f(0), 0]$. Or, equivalently, the exponential convergence of $\left[\tilde{u}, u_{t}\right]$ to $[0,0]$.

With this decomposition in mind, the large time behavior of all solutions in $X$ is completely determined by the corresponding behavior of solutions that take their initial data from

$$
V=\left\{[u, v] \in X: u(1)+\int_{0}^{1} \rho^{2} v d x=0\right\} .
$$

Note that $V$ is invariant under the flow given by (1.1) and is a closed subspace of $X$ in which the norm induced by the energy,

$$
\|[u, v]\|_{V}^{2} \equiv \int_{0}^{1}\left|u^{\prime}\right|^{2}+\rho^{2}|v|^{2} d x
$$

is equivalent to the one induced by $X$. 
We may now define the decay rate, as a function of $\rho$, as

$$
\omega(\rho)=\inf \left\{\omega: \exists C(\omega)>0 \text { such that } E(t) \leq C E(0) e^{2 \omega t},\right.
$$

for every solution of (1.1) with initial data in $V$ \}.

As in our study of the internally damped string, [2], we strive to identify $\omega(\rho)$ with the spectral abscissa of the matrix differential operator obtained on expressing (1.1) as the first order system $U_{t}=A U$. Here $U=\left[u, u_{t}\right]$, and $A: D(A) \rightarrow V$, is given by

$$
\begin{aligned}
A & =\left(\begin{array}{cc}
0 & I \\
\frac{1}{\rho^{2}} \frac{d^{2}}{d x^{2}} & 0
\end{array}\right) \\
D(A) & =\left\{[u, v] \in\left(H^{2}(0,1) \times H^{1}(0,1)\right) \cap V: u^{\prime}(0)=u^{\prime}(1)+v(1)=0\right\} .
\end{aligned}
$$

We shall see, assuming no more than (1.2), that $A$ possesses a compact dissipative inverse on $V$. As a result $\sigma(A)$, the spectrum of $A$, is composed of at most a countable number of eigenvalues, each an element of the left half plane. From $\sigma(A)$ we extract its spectral abscissa

$$
\mu(\rho)=\sup \{\Re \lambda: \lambda \in \sigma(A)\} .
$$

Our intuition is built on our understanding of $\mu(\rho)$ when $\rho$ is a fixed constant. If $U=[y, z] \in D(A)$ is an eigenvector of $A$ with eigenvalue $\lambda$ then $z=\lambda y$ and $y^{\prime \prime}=\lambda \rho^{2} z$, or

$$
y^{\prime \prime}=\lambda^{2} \rho^{2} y, \quad y^{\prime}(0)=y^{\prime}(1)+\lambda y(1)=0 .
$$

When $\rho$ is constant it follows that $y(x)=\cosh (\lambda \rho x)$ where $\lambda$ is determined by the right end condition, i.e., by $\lambda \rho \sinh (\lambda \rho)+\lambda \cosh (\lambda \rho)=0$, or

$$
\lambda \rho \tanh (\lambda \rho)=-\lambda .
$$

That $\lambda=0$ is not permitted follows from the fact that the associated eigenvector $U=[1,0] \notin V$. Hence, the eigenvalues are

$$
\begin{aligned}
\lambda_{n} & =-\frac{1}{\rho} \tanh ^{-1} \frac{1}{\rho} \\
& =-\frac{1}{2 \rho} \log \left|\frac{\rho+1}{\rho-1}\right|+i \frac{\pi}{\rho}\left\{\begin{array}{ll}
n & \text { if } \rho>1 \\
n+\frac{1}{2} & \text { if } \rho<1
\end{array} \quad n \in \mathbf{Z},\right.
\end{aligned}
$$


while the corresponding eigenvectors are

$$
U_{n}(x)=\cosh \left(\lambda_{n} \rho x\right)\left[1, \lambda_{n}\right] .
$$

We immediately note that $A$ has an empty spectrum when $\rho=1$ and that the spectral abscissa

$$
\mu(\rho)=-\frac{1}{2 \rho} \log \left|\frac{\rho+1}{\rho-1}\right|
$$

approaches $-\infty$ as $\rho \rightarrow 1$. If $\mu(\rho)$ and $\omega(\rho)$ are indeed one and the same the above suggests that the energy in the string of density one decays infinitely fast. This corresponds to the fact that, when $\rho=1$, the solutions to (1.1) "disappear" in finite time.

To see this, we express the wave equation as

$$
(\partial / \partial t-\partial / \partial x)\left(u_{t}+u_{x}\right)=0
$$

take into account the boundary condition at $x=1$, and find that $u_{t}+u_{x}=0$ for $0<x<1$ and $t>1-x$. Since $u_{x}(0, t)=0$ we deduce that $u_{t}(0, t)=0$ for $t \geq 1$. Thus $u_{t}(0, t)-u_{x}(0, t)=0$ for $t \geq 1$. Writing the wave equation as

$$
(\partial / \partial t+\partial / \partial x)\left(u_{t}-u_{x}\right)=0
$$

we conclude that $u_{t}-u_{x}=0$ for $0<x<1$ and $t>x+1$. Thus $u_{t}=u_{x}=0$ for $0<x<1$ and $t \geq 2$, i.e., $u$ is constant in time and space after time 2 .

If our goal is indeed to distribute mass along the string in such a way that the dissipative end is most effective, i.e., in such a way that energy decays at the greatest possible rate, then clearly the optimal choice is $\rho \equiv 1$. One could well stop here, declaring success, or, push on and work over a class of $\rho$ in which finite decay is assured. We shall push on, beginning with a short survey of previous related work on wave equations with a dissipative end condition.

Rideau [20], in considering

$$
\begin{aligned}
u_{t t}(x, t)-u_{x x}(x, t)=0, & 0<x<1,0<t, \\
u(0, t)=u_{x}(1, t)+a u_{t}(1, t)=0, & 0<t,
\end{aligned}
$$

found that the corresponding root vectors form a Riesz basis for $\left\{u_{1} \in H^{1}(0,1)\right.$ : $\left.u_{1}(0)=0\right\} \times L^{2}(0,1)$ when $a>0$ and $a \neq 1$. Cherkaoui [Ch] has recently extended this result to the case in which the right end condition takes the form

$$
u(0, t)=u_{x}(1, t)+a u_{t}(1, t)+b u(1, t)=0, \quad 0<t,
$$

where $b>0$. Working with constant coefficients Rideau and Cherkaoui exploit their explicit knowledge of the eigenvalues and eigenvectors. Recalling that a 
Riesz basis is, by definition, isomorphic to an orthonormal basis, it follows easily that the respective decay rates and spectral abscissas coincide in $[\mathrm{R}]$ and $[\mathrm{Ch}]$.

With respect to variable coefficient problems we remark that Krein and Nudelman in [11] and [12] considered (1.5) over a class of $\rho$ that permits measures, i.e., point masses. The first of these papers serves to announce the results proven in the second. The basic spectral theory for such generalized strings is developed in detail by Kac and Krein [7] and Dym and McKean [3]. Though Krein and Nudelman show that large eigenvalues obey no asymptotic formula for such $\rho$ they nonetheless establish that, so long as $\rho$ is not identically one on any interval of the form $(\ell, 1]$, the root vectors (when they exist) for (1.5) are complete in the weighted space $L^{2}\left(0,1 ; \rho^{2}\right)$. Recall that a collection of vectors is said to be complete when its linear span is dense.

Finally we note that (1.1) arises in the area of acoustic scattering concerned with the so-called Regge problem. Representatives of this literature that bear on our concerns include Hruščev, Nikol'skii, and Pavlov [6], Hruščev [5], and Shubov [22]. These authors approach (1.1) through the functional model of Nagy and Foias as developed, e.g., in Nikol'skii [17]. In particular, the result of [17, VI.5] is used to show that the eigenvectors associated with algebraically simple eigenvalues constitute a Riesz basis for their span. Appending to this a basis for the (finite dimensional) root space corresponding to the multiple eigenvalues one arrives at a Riesz basis for the entire space.

Our plan is as follows. In Section 2 we demonstrate that $\rho \equiv 1$ is the only choice of $\rho$ for which solutions of (1.1) vanish and so in all other cases $\sigma(A) \neq \varnothing$. In Section 4 we show that the eigenvectors of $A(\rho)$, when $\rho$ is constant, comprise a Riesz basis for $V$ and that the root vectors of $A(\rho)$, when $\rho$ is not one on any interval with right endpoint 1 , are complete in $V$. In Section 4 we establish sufficient conditions on $\rho$ for the existence and nonexistence of real eigenvalues of $A$. In Section 5 , under the assumption that $\rho$ is Lipschitz and $\rho(1) \neq 1$, we derive a lower bound on $\Re \lambda$ for $\lambda \in \sigma(A(\rho))$. Under the additional assumption that $\rho \in H^{2}(0,1)$ we, in Section 6 , construct precise asymptotic formulas for the large eigenvalues and eigenvectors of $A$. These formulas lead immediately, in Section 7, to the conclusion that the associated root vectors comprise a Riesz basis for $V$ and, as such, $\omega(\rho)=\mu(\rho)$. The requirement that $\rho$ lie in $H^{2}(0,1)$ is essentially one of convenience. It will be clear from Section 6 that one need only assume that $\rho^{\prime}$ has finite total variation.

Our use of Livšic's criterion to establish completeness and Bari's to arrive at a Riesz basis culminates in a much more direct approach than that advocated by [6], [5], and [22].

With the identification of $\omega(\rho)$ and $\mu(\rho)$ the minimization of the former reduces to the problem of forcing the spectrum of $A(\rho)$ as far left as possible. In Section 8 , we show that $\omega$ achieves its minimum over

$$
a d=\left\{\rho: 0<\alpha \leq \rho(x),|\rho(1)-1| \geq \gamma>0,\|\rho\|_{H^{2}} \leq C\right\} .
$$


We close in Section 9 with comments on the corresponding multidimensional problem.

\section{The Existence of Eigenvalues}

The eigenvalues of $A$ are the poles of the resolvent $\lambda \mapsto(A-\lambda)^{-1}$. To solve $(A-\lambda)\left[v_{1}, v_{2}\right]=\left[f_{1}, f_{2}\right]$ in $V$ is to set $v_{2}=\lambda v_{1}+f_{1}$ and solve

$$
\begin{aligned}
& v_{1}^{\prime \prime}-\lambda^{2} \rho^{2} v_{1}=\rho^{2}\left(\lambda f_{1}+f_{2}\right) \\
& v_{1}^{\prime}(0)=0, \quad v_{1}^{\prime}(1)+\lambda v_{1}(1)+f_{1}(1)=0,
\end{aligned}
$$

subject to

$$
v_{1}(1)=-\lambda \int_{0}^{1} \rho^{2} v_{1} d x-\int_{0}^{1} \rho^{2} f_{1} d x
$$

We note that $\frac{1}{2} f_{1}(1)\left(1-x^{2}\right)$ satisfies the boundary conditions in (2.1) and so write $v_{1}(x)=\frac{1}{2} f_{1}(1)\left(1-x^{2}\right)+w(x)$ where $w$ must now solve

$$
\begin{gathered}
w^{\prime \prime}-\lambda^{2} \rho^{2} w=\rho^{2}\left(\lambda f_{1}+f_{2}+\frac{1}{2} \lambda^{2} f_{1}(1)\left(1-x^{2}\right)\right)+f_{1}(1), \\
w^{\prime}(0)=w^{\prime}(1)+\lambda w(1)=0 .
\end{gathered}
$$

We first show that this problem has a one parameter family of solutions when $\lambda=0$ and that (2.2) selects a particular one. When $\lambda=0$ we find

$$
w^{\prime \prime}=\rho^{2} f_{2}+f_{1}(1), \quad w^{\prime}(0)=w^{\prime}(1)=0 .
$$

The solvability condition, $\int_{0}^{1} \rho^{2} f_{2} d x+f_{1}(1)=0$, that stems from the Fredholm Alternative exactly coincides with one of the requirements for $\left[f_{1}, f_{2}\right] \in V$. As a result,

$$
w(x)=a+\int_{0}^{x}(x-s)\left(\rho^{2}(s) f_{2}(s)+f_{1}(1)\right) d s, \quad a \in \mathbf{R} .
$$

We choose $a$ to satisfy (2.2), i.e., $w(1)=-\int_{0}^{1} \rho^{2} f_{1} d x$, and so arrive at

$$
v_{1}(x)=\int_{0}^{x}(x-s) \rho^{2} f_{2} d s-\int_{0}^{1}(1-s) \rho^{2} f_{2} d s-\int_{0}^{1} \rho^{2} f_{1} d s .
$$

Noting that $v_{2}=f_{1}$ when $\lambda=0$, we summarize the above in

$$
A^{-1}\left[f_{1}, f_{2}\right]=\left[\int_{0}^{x}(x-s) \rho^{2} f_{2} d s-\int_{0}^{1}(1-s) \rho^{2} f_{2} d s-\int_{0}^{1} \rho^{2} f_{1} d s, f_{1}\right] .
$$


From the boundedness of $\rho$ and the compactness of the imbedding of $H^{1}(0,1)$ in to $L^{2}(0,1)$ follows the compactness of $A^{-1}$ on $V$. As a result, the spectrum of $A$ is composed of at most a countable number of eigenvalues, $\left\{\lambda_{k}\right\}_{k}$.

We now return to $(2.3)$, assume $\lambda \neq 0$, and characterize the $\lambda_{k}$ as the zeros of a shooting function. In particular, we introduce $\varphi(x, \lambda)$ and $\psi(x, \lambda)$, solutions of the respective initial and terminal value problems,

$$
\begin{aligned}
& \varphi^{\prime \prime}-\lambda^{2} \rho^{2} \varphi=0, \quad \varphi(0, \lambda)=1, \varphi^{\prime}(0, \lambda)=0 \\
& \psi^{\prime \prime}-\lambda^{2} \rho^{2} \psi=0, \quad \psi(1, \lambda)=1 / \lambda, \psi^{\prime}(1, \lambda)=-1 .
\end{aligned}
$$

We note that $\varphi$ likewise satisfies the integral equation

$$
\varphi(x, \lambda)=1+\lambda^{2} \int_{0}^{x}(x-s) \rho^{2}(s) \varphi(s, \lambda) d s,
$$

and denote the corresponding shooting function by

$$
Q(\lambda) \equiv \varphi^{\prime}(1, \lambda) / \lambda+\varphi(1, \lambda)
$$

Clearly $\sigma(A)$ coincides with the set of zeros of $Q$. In the introduction we found that if $\rho \equiv 1$ then $Q$ never vanishes and $\sigma(A)=\varnothing$. We now show that to be the only pathological case.

Theorem 2.1. If $\rho$ satisfies (1.2) and $\rho$ is not identically one then $\sigma(A) \neq$ $\varnothing$.

Proof. Dym and McKean [3, Section 6.3(6)] demonstrate that $Q$ is of exponential type $\int_{0}^{1} \rho d x$, i.e.,

$$
\int_{0}^{1} \rho d x=\limsup _{R \uparrow \infty} R^{-1} \max _{\vartheta \in[0,2 \pi)} \log \left|Q\left(R e^{i \vartheta}\right)\right| .
$$

It then follows from Hadamard's Factorization Theorem that

$$
Q(\lambda)=e^{a \lambda} \prod_{n}\left(1-\lambda / \lambda_{n}\right) e^{\lambda / \lambda_{n}}
$$

where $\left\{\lambda_{n}\right\}_{n}$ is the zero set of $Q$ and $a$ is a complex constant. If this zero set is empty then the product defaults to one and $Q(\lambda)=e^{a \lambda}$. As $Q(\lambda)$ is real for real $\lambda$ it follows that $a$ is real and in fact $a=\int_{0}^{1} \rho d x$. We now deduce the restraints 
this places on $\varphi$. We follow Kac and Krein [7, Section 2] and develop $\varphi$, as the solution of (2.7), in powers of $\lambda^{2}$,

$$
\varphi(x, \lambda)=\sum_{n=0}^{\infty} \varphi_{n}(x) \lambda^{2 n}, \quad \varphi_{0}(x) \equiv 1, \quad \varphi_{j+1}(x)=\int_{0}^{x}(x-s) \rho^{2} \varphi_{j} d s .
$$

It follows that $\lambda \mapsto \varphi(1, \lambda)$ and $\lambda \mapsto \varphi^{\prime}(1, \lambda) / \lambda$ are power series in $\lambda^{2}$ and $\lambda$ respectively. As $Q(\lambda)=e^{a \lambda}=\cosh (a \lambda)+\sinh (a \lambda)$ we find the explicit representations

$$
\varphi(1, \lambda)=\cosh (a \lambda) \quad \text { and } \quad \varphi^{\prime}(1, \lambda)=\lambda \sinh (a \lambda) .
$$

We recognize the former as the shooting function for the Neumann-Dirichlet problem

$$
\eta^{\prime \prime}=\nu^{2} \rho^{2} \eta, \quad \eta^{\prime}(0)=\eta(1)=0,
$$

and the latter as the shooting function for the Neumann-Neumann problem

$$
\zeta^{\prime \prime}=\chi^{2} \rho^{2} \zeta, \quad \zeta^{\prime}(0)=\zeta^{\prime}(1)=0 .
$$

In particular, from (2.11) it follows that

$$
\nu_{n}=\frac{i \pi}{2 a}(2 n-1) \quad \text { and } \quad \chi_{n}=\frac{i \pi}{a}(n-1), \quad n=1,2, \ldots
$$

On recalling the well-known fact, see, e.g., [3, Section 6.6], that two such spectra uniquely determine a bounded $\rho$ we immediately conclude that $\rho$ is identically $a$. Hence, it suffices to restrict ourselves to constant $\rho$. From the introduction we now recall that $Q(\lambda)=e^{\rho \lambda}$ if and only if $\rho=1$.

We recall that Majda [15] has shown that no solution of

$$
\begin{aligned}
u_{t t}-\Delta u+c(x) u_{t} & =0, \quad x \in \Omega, t>0, \\
\frac{\partial u}{\partial n}+\gamma(x) u_{t} & =0, \quad x \in \partial \Omega, t>0,
\end{aligned}
$$

disappears when (i) $\Omega$ is bounded in $\mathbf{R}^{n}$, (ii) $\partial \Omega$ and $\gamma$ are real analytic, (iii) $\gamma(x) \neq 1 \forall x \in \partial \Omega$, and (iv) $\operatorname{supp} c(x) \cap \partial \Omega=\varnothing$.

If $\lambda_{n} \in \sigma(A)$ we note that as a consequence of the uniqueness of $\varphi\left(1, \lambda_{n}\right)$ that each eigenvector of $A$ corresponding to $\lambda_{n}$ must be a scalar multiple of $U_{n}(x) \equiv$ $\varphi\left(x, \lambda_{n}\right)\left[1, \lambda_{n}\right]$. In other words, the geometric multiplicity of each eigenvalue is one. As a result, the algebraic multiplicity of an eigenvalue is its order as pole of $(A-\lambda)^{-1}$. We now associate this order with $Q$. 
If $\lambda \notin \sigma(A)$ then (2.3) has a unique solution that is consistent with (2.2). In particular,

$$
w(x)=G(\lambda)\left\{\rho^{2}\left(\lambda f_{1}+f_{2}+\frac{1}{2} \lambda^{2} f_{1}(1)\left(1-x^{2}\right)\right)+f_{1}(1)\right\},
$$

where $G(\lambda)$ is the Green's operator

$$
G(\lambda) \eta(x)=\int_{0}^{1} g(x, \xi, \lambda) \eta(\xi) d \xi
$$

where

$$
g(x, \xi, \lambda)= \begin{cases}\frac{\psi(x, \lambda) \varphi(\xi, \lambda)}{Q(\lambda)} & \text { if } \quad 0 \leq \xi \leq x \leq 1 \\ \frac{\psi(\xi, \lambda) \varphi(x, \lambda)}{Q(\lambda)} & \text { if } 0 \leq x \leq \xi \leq 1 .\end{cases}
$$

Hence the algebraic multiplicity of $\lambda_{n} \in \sigma(A)$ is its order as a zero of $Q$.

Some preliminary information is obtained upon taking the $L^{2}(0,1)$ inner product of $(2.5)$ at $\lambda=\lambda_{n}$ with $\varphi_{n}(x) \equiv \varphi\left(x, \lambda_{n}\right)$. One finds

$$
\lambda_{n}^{2} \int_{0}^{1} \rho^{2}\left|\varphi_{n}\right|^{2} d x+\lambda_{n}\left|\varphi_{n}(1)\right|^{2}+\int_{0}^{1}\left|\varphi_{n}^{\prime}\right|^{2} d x=0
$$

and therefore

$$
\lambda_{n}=\frac{-\left|\varphi_{n}(1)\right|^{2} \pm \sqrt{\left|\varphi_{n}(1)\right|^{4}-4 \int_{0}^{1} \rho^{2}\left|\varphi_{n}\right|^{2} d x \int_{0}^{1}\left|\varphi_{n}^{\prime}\right|^{2} d x}}{2 \int_{0}^{1} \rho^{2}\left|\varphi_{n}\right|^{2} d x} .
$$

It follows that $\Re \lambda_{n} \leq 0$, in fact $\Re \lambda_{n}<0$, for equality would force $\varphi_{n}(1)=$ $\varphi_{n}^{\prime}(1)=0$ and hence $\varphi_{n} \equiv 0$. Dym and McKean [3, Section 6.3] also show that

$$
\int_{-\infty}^{\infty} \frac{\log _{+}|Q(-i x)|}{1+x^{2}} d x<\infty
$$

from which it follows, see, e.g., Levin [14, Section V.4, Theorem 11], that there exist $C_{1}$ and $C_{2}$ for which

$$
\left|\Re \lambda_{n}\right| \leq C_{1}+C_{2}\left|\Im \lambda_{n}\right|
$$

Majda [16], in the context of (2.12), has bettered this with

$$
\left|\Re \lambda_{n}\right| \leq C_{1}+C_{2}\left|\Im \lambda_{n}\right|^{3 / 4} \text {. }
$$

We shall soon see in fact that $\left|\Re \lambda_{n}\right| \leq C_{1}$ when $\rho$ is Lipschitz.

\section{The Completeness of the Root Vectors}

We follow Krein and Nudelman [11] in their application of the following result of Livšic. We assume throughout that $\rho$ is not identically one. 
Theorem 3.1 ([10, Section 2.5]). If $H$ is Hilbert and $T: H \rightarrow H$ is linear and compact and $T_{\Re} \equiv \frac{1}{2}\left(T+T^{*}\right)$ is nonpositive and of finite trace then

$$
\sum_{\nu_{n} \in \sigma(T)}\left|\Re \nu_{n}\right| \leq-\operatorname{tr}\left(T_{\Re}\right)
$$

where the $\nu_{n}$ are repeated according to their algebraic multiplicity. Equality holds in (3.1) if and only if the root vectors of $T$ are complete in $H$.

We note that $\left(T^{*}\right)_{\Re}=T_{\Re}$ and that if $T$ is real then $\sigma\left(T^{*}\right)=\sigma(T)$ including algebraic multiplicities. Hence, if $T$ is real and equality holds in (3.1) then the root vectors of $T^{*}$ are complete in $H$ as well.

We show that $\left(A^{-1}\right)_{\Re}$ is nonpositive and of rank one. In particular,

$$
\begin{aligned}
\left(A^{-1}\right)^{*}\left[f_{1}, f_{2}\right]=\left[\int_{0}^{1}(1-s) \rho^{2} f_{2} d s-\int_{0}^{x}(x-s) \rho^{2} f_{2} d s+\int_{0}^{1} \rho^{2} f_{1} d s\right. \\
\left.+2 \int_{0}^{1} \rho^{2} d s \int_{0}^{1} \rho^{2} f_{2} d s,-f_{1}-2 \int_{0}^{1} \rho^{2} f_{2} d s\right]
\end{aligned}
$$

and so

$$
\left(A^{-1}\right)_{\Re}\left[f_{1}, f_{2}\right]=\int_{0}^{1} \rho^{2} f_{2} d x\left[\int_{0}^{1} \rho^{2} d x,-1\right]
$$

is indeed rank-one while

$$
\left\langle\left(A^{-1}\right)_{\Re}\left[f_{1}, f_{2}\right],\left[f_{1}, f_{2}\right]\right\rangle=-\left|\int_{0}^{1} \rho^{2} f_{2} d s\right|^{2}
$$

and

$$
\operatorname{tr}\left(A^{-1}\right)_{\Re}=-\int_{0}^{1} \rho^{2} d x .
$$

From Theorem 3.1 we may now draw the following result:

Corollary 3.2. If $\rho$ satisfies (1.2), then

$$
\sum_{\lambda_{n} \in \sigma(A)} \frac{\left|\Re \lambda_{n}\right|}{\left|\lambda_{n}\right|^{2}} \leq \int_{0}^{1} \rho^{2} d x .
$$

We now produce conditions under which equality holds in Corollary 3.2. In the constant case we offer the following elementary argument. 
Theorem 3.3. If $\rho$ is a positive constant distinct from one then the $\lambda_{n} \in \sigma(A)$, see (1.6), are each of algebraic multiplicity one and their associated eigenvectors are complete in $V$.

Proof. We assume $\rho>1$. The other case being similar. We sum the $\left|\Re \lambda_{n}\right| /\left|\lambda_{n}\right|^{2}$ without repetition,

$$
\sum_{n} \frac{\left|\Re \lambda_{n}\right|}{\left|\lambda_{n}\right|^{2}}=\frac{\rho}{\nu}+2 \nu \rho \sum_{n=1}^{\infty} \frac{1}{\nu^{2}+n^{2} \pi^{2}}, \quad \nu \equiv \frac{1}{2} \log \left(\frac{\rho+1}{\rho-1}\right) .
$$

As $\nu^{2}+n^{2} \pi^{2}$ is the $n$th eigenvalue of $L u \equiv-u^{\prime \prime}+\nu^{2} u, u \in H_{0}^{1}(0,1)$, it follows from the standard trace formula that

$$
\sum_{n=1}^{\infty} \frac{1}{\nu^{2}+n^{2} \pi^{2}}=\int_{0}^{1} g(x, x) d x
$$

where

$$
g(x, y)=\frac{\sinh \nu(x \vee y) \sinh \nu(1-(x \wedge y))}{\nu \sinh \nu}
$$

is the Green's function for $L$. Hence,

$$
\sum_{n=1}^{\infty} \frac{1}{\nu^{2}+n^{2} \pi^{2}}=\frac{\nu \cosh \nu-\sinh \nu}{2 \nu^{2} \sinh \nu}=\frac{\rho}{2 \nu}-\frac{1}{2 \nu^{2}} .
$$

inserting this sum in (3.2) we find that equality holds in Corollary 3.2.

The fact that a (bi)normalized copy of $\left\{U_{n}\right\}_{n}$ for constant $\rho$ is in fact a Riesz basis for $V$ now follows easily. As in [2] our principal tool is the following result of Bari.

Theorem 3.4 [4, Theorem 2.1, Chapter VI]. $\left\{f_{n}\right\}_{n}$ is a Riesz basis for the Hilbert space $H$ if and only if $\left\{f_{n}\right\}_{n}$ is complete in $H$ and there corresponds to it a complete biorthogonal sequence $\left\{g_{n}\right\}_{n}$, and for any $f \in H$ both $\left\{\left\langle f_{n}, f\right\rangle\right\}_{n}$ and $\left\{\left\langle g_{n}, f\right\rangle\right\}_{n}$ are square summable.

We shall also make use of the equivalent statement that $\left\{f_{n}\right\}_{n}$ is a Riesz basis for $H$ iff $\left\{f_{n}\right\}_{n}$ is complete and there exist two constants $c_{0}$ and $c_{1}$ such

$$
c_{0} \sum_{n=1}^{N}\left|a_{n}\right|^{2} \leq\left\|\sum_{n=1}^{N} a_{n} f_{n}\right\|_{H}^{2} \leq c_{1} \sum_{n=1}^{N}\left|a_{n}\right|^{2}
$$

for each $N$ and each $\left\{a_{n}\right\}_{n} \in \mathbf{C}^{N}$. 
The sequence biorthogonal to $\left\{U_{n}\right\}_{n}$ is built from the eigenvectors of the adjoint of $A$,

$$
\begin{aligned}
A^{*} & =-\left(\begin{array}{cc}
0 & I \\
\frac{1}{\rho^{2}} \frac{d^{2}}{d x^{2}} & 0
\end{array}\right) \\
D\left(A^{*}\right) & =\left\{[u, v] \in\left(H^{2}(0,1) \times H^{1}(0,1)\right) \cap V: u^{\prime}(0)=u^{\prime}(1)-v(1)=0\right\} .
\end{aligned}
$$

We note that $\sigma\left(A^{*}\right)=\sigma(A)$ and that if $W_{k}$ is an eigenvector of $A^{*}$ corresponding to $\bar{\lambda}_{k}$ then, for constant $\rho, W_{k}(x)=\cosh \left(\bar{\lambda}_{k} \rho x\right)\left[-1, \bar{\lambda}_{k}\right]$ and $\left\langle U_{j}, W_{k}\right\rangle=\lambda_{k}^{2} \rho^{2} \delta_{j k}$. As a result

$$
\begin{gathered}
\tilde{U}_{k}(x)=\frac{1}{\lambda_{k} \rho} \cosh \left(\lambda_{k} \rho x\right)\left[1, \lambda_{k}\right], \\
\tilde{W}_{k}(x)=\frac{1}{\bar{\lambda}_{k} \rho} \cosh \left(\bar{\lambda}_{k} \rho x\right)\left[-1, \bar{\lambda}_{k}\right]
\end{gathered}
$$

satisfy $\left\langle\tilde{U}_{j}, \tilde{W}_{k}\right\rangle=\delta_{j k}$, i.e., they constitute a biorthogonal set in $V$. That $\left\{\tilde{W}_{n}\right\}_{n}$ is complete in $V$ follows from the remark following Theorem 3.1. It remains to select $\left[f_{1}, f_{2}\right] \in V$ and check $\left\{\left\langle\tilde{U}_{n},\left[f_{1}, f_{2}\right]\right\rangle\right\} \in \ell^{2}(\mathbf{C})$. We suppose $\rho>1$, the other case being similar, and recall the definition of $\nu$ in (3.2). That

$$
\begin{aligned}
\left\langle\tilde{U}_{n},\left[f_{1}, f_{2}\right]\right\rangle= & \int_{0}^{1} \sinh \left(\lambda_{n} \rho x\right) \bar{f}_{1}^{\prime}+\rho \cosh \left(\lambda_{n} \rho x\right) \bar{f}_{2} d x \\
= & \int_{0}^{1}\left(\sinh (\nu) \bar{f}_{1}^{\prime}+\rho \cosh (\nu) \bar{f}_{2}\right) \cos (n \pi x) d x \\
& \quad+i \int_{0}^{1}\left(\cosh (\nu) \bar{f}_{1}^{\prime}+\rho \sinh (\nu) \bar{f}_{2}\right) \sin (n \pi x) d x
\end{aligned}
$$

is square summable now follows directly from $\left[f_{1}, f_{2}\right] \in V$. The verification that $\left\{\left\langle\tilde{W}_{n},\left[f_{1}, f_{2}\right]\right\rangle\right\}_{n} \in \ell^{2}(\mathbf{C})$ is just as simple. From Theorem 3.4. we may now deduce the following.

Theorem 3.5. If $\rho$ is a positive constant distinct from one then the eigenvectors $\left\{\tilde{U}_{n}\right\}_{n}$, see (3.4), constitute a Riesz basis for $V$. If we append to this sequence the vector $[1,0]$, corresponding to the zero eigenvalue of $A$ in $X$, we obtain a Riesz basis for $X$. 
We now return to the variable coefficient case and address the extent to which the root vectors of $A$ are complete in $V$. In particular, we return to [11] and equate the power series representation of $Q$ stemming from (2.10) with a refinement of (2.9). First, it follows directly from (2.8) and (2.10) that

$$
Q(\lambda)=1+\lambda \int_{0}^{1} \rho^{2} d x+O\left(\lambda^{2}\right) .
$$

Next, from the summability of $\left|\Re \lambda_{n}\right| /\left|\lambda_{n}\right|^{2}$ comes the fact that one may remove the exponential factors in (2.9), i.e.,

$$
Q(\lambda)=e^{a \lambda} \prod_{\Im \lambda_{n}>0}\left(1-\frac{\lambda}{\lambda_{n}}\right)\left(1-\frac{\lambda}{\bar{\lambda}_{n}}\right) \prod_{\Im \lambda_{n}=0}\left(1-\frac{\lambda}{\lambda_{n}}\right) .
$$

See [3, Section 6.2] for an independent proof. From the easily verified fact that if $\rho(x)=1$ for $x \in(\ell, 1]$ then $\sigma(A)$ coincides with the spectrum associated with (1.1) for $x \in(0, \ell)$ subject to $u_{x}(0, t)=u_{x}(\ell, t)+u_{t}(\ell, t)=0$. Krein and Nudelman next deduce that $a$ is in fact the largest number $b$ for which $\rho$ is identically one on the interval $(1-b, 1)$. Identifying the coefficients of $\lambda$ in (3.5) and (3.6) we find

$$
\int_{0}^{1} \rho^{2} d x=a-\sum_{\lambda_{n} \in \sigma(A)} \frac{\Re \lambda_{n}}{\left|\lambda_{n}\right|^{2}} .
$$

As a result, analogous to [11, Theorem 2], we have the following.

Theorem 3.6. If $\rho$ satisfies (1.2) then the root vectors of $A$ are complete in $V$ if and only if $\rho$ is not identically one on any interval of the form $(\ell, 1)$.

The proof that these root vectors indeed make up a basis for $V$ will require a considerably more detailed study of the $\lambda_{n}$. In the next three sections we respectively analyze the real eigenvalues, establish a crude lower bound on $\Re \lambda_{n}$, and develop asymptotic estimates for $\lambda_{n}$.

\section{Real Eigenvalues}

Recall that when $\rho$ is constant there exists either one or zero real eigenvalues depending on whether $\rho$ is greater than or less than one. In the variable coefficient case, with respect to (1.2), we prove the absence of real eigenvalues when $\beta<1$ and the presence of at least one real eigenvalue when $\alpha>1$. In addition we produce explicit upper and lower bounds. One such upper bound follows immediately from Corollary 3.2. In particular, if $\lambda(\rho)$ is a real eigenvalue of $A$ then

$$
\lambda(\rho)<\frac{-1}{\int_{0}^{1} \rho^{2} d x} .
$$


In order to proceed we shall find it convenient to demote the $\lambda$ appearing in the boundary condition of (1.5) to the status of a parameter. More precisely, we consider the regular Sturm-Liouville problem,

$$
\eta^{\prime \prime}=\nu \rho^{2} \eta, \quad \eta^{\prime}(0)=\eta^{\prime}(1)+\lambda \eta(1)=0,
$$

for fixed negative $\lambda$. We label its eigenvalues

$$
\nu_{1}(\lambda)>\nu_{2}(\lambda)>\nu_{3}(\lambda)>\cdots \rightarrow-\infty,
$$

denote by $\left\{\eta_{k}\right\}_{k}$ the corresponding orthonormal base of eigenfunctions, and note that $\lambda$ is a real eigenvalue of $A$ iff $\lambda$ is a solution of

$$
\nu_{k}(\lambda)=\lambda^{2},
$$

for some $k$. We now show that (4.2) has no solutions for $k \neq 1$.

Theorem 4.1. If $\lambda<0$ then $\nu_{1}(\lambda)>0>\nu_{2}(\lambda)$ and $\eta_{1}$ is positive, convex, and strictly increasing. Hence, $\left\|\eta_{1}\right\|_{\infty}=\eta_{1}(1)$.

Proof. $\eta_{1}$ is clearly of one sign. Without loss we assume it positive. As a result $\eta_{1}^{\prime \prime}$ has the sign of $\nu_{1}$. If $\nu_{1}<0$ then $\eta_{1}$ is concave and $\eta_{1}^{\prime}(1)<0$. Hence, $\eta_{1}^{\prime}(1)+\lambda \eta_{1}(1)<0$, contrary to (4.1). So $\nu_{1}(\lambda)>0$ and $\eta_{1}$ is convex. As $\eta_{1}^{\prime}(0)=0$ it follows that $\eta_{1}$ is strictly increasing.

We suppose, without loss, that $\eta_{2}(x)>0$ for $x \in(0, \xi)$. As $\eta_{2}^{\prime}(0)=0$ it follows that $\eta_{2}$ must be concave on a subinterval of $(0, \xi)$. It follows that $\nu_{2}(\lambda)<0$.

Hence it suffices to establish the solvability of the single equation $\nu_{1}(\lambda)=\lambda^{2}$.

Theorem 4.2. (i) If $\beta<1$ then $A$ has no real eigenvalues. (ii) If $\alpha>1$ then $A$ has at least one real eigenvalue. In this case each real eigenvalue satisfies the lower bound

$$
-\frac{1}{2 \alpha} \log \left(\frac{\alpha+1}{\alpha-1}\right)<\lambda(\rho)
$$

Proof. We exploit the variational characterization

$$
\nu_{1}(\lambda)=\max _{\eta \in H^{1}(0,1)} \frac{-\lambda \eta^{2}(1)-\int_{0}^{1}\left|\eta^{\prime}\right|^{2} d x}{\int_{0}^{1} \rho^{2} \eta^{2} d x} .
$$

As a maximum of affine functions of $\lambda$ it follws that $\lambda \mapsto \nu_{1}(\lambda)$ is convex and hence continuous. This characterization also permits us to bound $\nu_{1}(\lambda)$ in terms 
of its values in the extreme case when $\rho$ is either identically $\alpha$ or $\beta$. In particular, $\nu_{1}(\lambda, \beta) \leq \nu_{1}(\lambda, \rho) \leq \nu_{1}(\lambda, \alpha)$. A simple calculation reveals $\nu_{1}(\lambda, \beta)$ to be the positive solution of $\beta \sqrt{\nu} \tanh \beta \sqrt{\nu}=-\lambda$. As $|\tanh x| \leq 1$ we note that $\nu_{1}(\lambda, \beta)$ is greater than the solution of $\beta \sqrt{\nu}=-\lambda$. Hence,

$$
\nu_{1}(\lambda, \rho)>\lambda^{2} / \beta^{2},
$$

and so, if $\beta<1,(4.2)$ has no solutions. Part (i) is now proved.

In a similar fashion, we find that $\alpha \sqrt{\nu} \tanh \alpha \sqrt{\nu}>\sqrt{\nu}$ when $\alpha>1$ and

$$
\sqrt{\nu}>\frac{1}{2 \alpha} \log \left(\frac{\alpha+1}{\alpha-1}\right) \text {. }
$$

As a result, $\nu_{1}(\lambda, \rho)<\lambda^{2}$ when $-\lambda>\frac{1}{2 \alpha} \log \left(\frac{\alpha+1}{\alpha-1}\right)$. To complete the proof of (ii) it remains to show that $\nu_{1}(\lambda, \rho)>\lambda^{2}$ for small $-\lambda$. That such is the case follows on choosing $\eta \equiv 1$ in (4.3) and finding

$$
\nu_{1}(\lambda) \geq \frac{-\lambda}{\int_{0}^{1} \rho^{2} d x} .
$$

Part (ii) is proved upon recalling the continuity of $\lambda \mapsto \nu_{1}(\lambda)$.

\section{A Lower bound on $\Re \lambda_{n}$}

We here show that if $\rho$ is Lipschitz and $\rho(1) \neq 1$ then $A(\rho)$ is a bounded perturbation of the constant coefficient operator $A(\rho(1)) \equiv A_{1}$. This enables us to bound the distance between $\sigma(A(\rho))$ and $\sigma\left(A_{1}\right)$.

Let us introduce the abbreviations,

$$
m \equiv \int_{0}^{1} \rho d x \quad \text { and } \quad \rho_{1} \equiv \rho(1)
$$

and the new variables

$$
\xi(x)=\frac{1}{m} \int_{0}^{x} \rho(s) d s \quad \text { and } \quad \tau=\frac{\rho_{1}}{m} t .
$$

In these variables (1.1) becomes

$$
\begin{gathered}
\rho_{1}^{2} u_{\tau \tau}(\xi, \tau)-u_{\xi \xi}(\xi, \tau)-b(\xi) u_{\xi}(\xi, \tau)=0, \quad 0<\xi<1,0<\tau, \\
u_{\xi}(0, \tau)=u_{\xi}(1, \tau)+u_{\tau}(1, \tau)=0,0<\tau,
\end{gathered}
$$


where

$$
b(\xi)=\frac{m \rho^{\prime}(x(\xi))}{\rho^{2}(x(\xi))} .
$$

The associated first order system takes the form

$$
\begin{aligned}
U_{t} & =\left(\begin{array}{cc}
0 & I \\
\frac{1}{\rho_{1}^{2}} \frac{d^{2}}{d \xi^{2}} & 0
\end{array}\right) U+\left(\begin{array}{cc}
0 & 0 \\
b \frac{d}{d \xi} & 0
\end{array}\right) U \\
& \equiv A_{1} U+B U,
\end{aligned}
$$

where $D\left(A_{1}\right)=\left\{[u, v] \in H^{2}(0,1) \times H^{1}(0,1): u^{\prime}(0)=u^{\prime}(1)+v(1)=0\right\}$ and $B \in L(X)$, the space of bounded linear operators on $X=H^{1}(0,1) \times L^{2}(0,1)$. In particular,

$$
\begin{aligned}
\|B\|_{L(X)} & =\sup _{\|[u, v]\|_{X}=1}\|B[u, v]\|_{X}=\sup _{\|u\|_{H^{1}}=1}\left\|b u^{\prime}\right\|_{2} \\
& \leq\|b\|_{\infty} \leq \frac{m}{\alpha^{2}}\left\|\rho^{\prime}\right\|_{\infty} .
\end{aligned}
$$

This bound, together with the fact that the eigenvectors $\left\{U_{n}\right\}_{n}$, of $A_{1}$ comprise a Riesz basis for $X$, now permits us to bound the distance between $\sigma(A(\rho))$ and $\sigma\left(A_{1}\right)$. In particular, if $\lambda$ is an eigenvalue of $A_{1}+B$ with eigenvector $U \in X$ then this $U$ has the expansion

$$
U=\sum_{n} a_{n} U_{n}
$$

and so

$$
B U=\lambda U-A_{1} U=\sum_{n} a_{n}\left(\lambda-\Lambda_{n}\right) U_{n},
$$

where $\left\{\Lambda_{n}\right\}_{n}=\sigma\left(A_{1}\right)$. Recalling (3.3) we find

$$
c_{0} \sum_{n}\left|\lambda-\Lambda_{n}\right|^{2}\left|a_{n}\right|^{2} \leq\left\|\lambda U-A_{1} U\right\|_{X}^{2}=\|B U\|_{X}^{2} \leq\|B\|_{L(X)}^{2} c_{1} \sum_{n}\left|a_{n}\right|^{2} .
$$

As $U \neq 0$ it follows that there exists a $k \in \mathbf{Z}$ for which

$$
\left|\lambda-\Lambda_{k}\right|^{2} \leq \frac{c_{1}}{c_{0}}\|B\|_{L(X)}^{2},
$$

or, equivalently,

$$
\operatorname{dist}\left(\sigma(A), \sigma\left(A_{1}\right)\right) \leq \sqrt{\frac{c_{1}}{c_{0}}}\|B\|_{L(X)} .
$$

We note that $\sqrt{c_{1} / c_{0}}$ is the condition number of the isomorphism of $\ell^{2}(\mathbf{C})$ with the matrix representation $\left\langle U_{j}, U_{k}\right\rangle_{X}$. Restricting ourselves to real parts we note that (5.3) and (5.2) establish 
Theorem 5.1. If, in addition to (1.2), $\rho$ is Lipschitz and $\rho_{1} \neq 1$ then if $\lambda_{n} \in \sigma(A(\rho))$ then

$$
\Re \lambda_{n} \geq-\frac{1}{2 \rho_{1}} \log \left|\frac{\rho_{1}+1}{\rho_{1}-1}\right|-\sqrt{\frac{c_{1}}{c_{0}}} \frac{m}{\alpha^{2}}\left\|\rho^{\prime}\right\|_{\infty}
$$

For future reference, let us abbreviate this right hand side by $-\kappa$.

\section{High Frequencies}

We now develop asymptotic formulas for $\lambda_{n}$ and $U_{n}$ as $\left|\lambda_{n}\right| \rightarrow \infty$. Our development can be seen both as an elaboration of [6], where few details are provided and more function theory is invoked, and as a special case of [22], where, in her desire to permit $\rho$ to either vanish or become infinite at the damped end, Shubov requires a twenty page immersion in special functions. At the heart of both approaches, as with our previous work [2], is a fake potential that has the advantage that its introduction into (2.6) permits one to find an explicit solution. One then argues that the fake potential has a negligible effect on the high frequencies. The fake potential in this case

$$
q(x) \equiv \rho^{1 / 2}(x)\left(\rho^{-1 / 2}(x)\right)^{\prime \prime}=\frac{3}{4}\left(\frac{\rho^{\prime}(x)}{\rho(x)}\right)^{2}-\frac{\rho^{\prime \prime}(x)}{2 \rho(x)}
$$

lies in $L^{2}(0,1)$ so long as, in addition to $(1.2), \rho \in H^{2}(0,1)$. Its addition to (2.6) brings us to

$$
z^{\prime \prime}-q z=\lambda^{2} \rho^{2} z, \quad z(1)=\lambda^{-1}, z^{\prime}(1)=-1,
$$

the solution to which is

$$
\begin{aligned}
z(x, \lambda) & =\frac{\rho_{1} \cosh \left(\lambda \int_{x}^{1} \rho d s\right)+\sinh \left(\lambda \int_{x}^{1} \rho d s\right)}{\lambda \sqrt{\rho_{1} \rho(x)}}-\frac{(\log \rho)^{\prime}(1) \sinh \left(\lambda \int_{x}^{1} \rho d s\right)}{2 \lambda^{2} \sqrt{\rho_{1} \rho(x)}} \\
& \equiv w_{1}(x, \lambda)+O\left(|\lambda|^{-2}\right) .
\end{aligned}
$$

Recall that in $(5.1)$ we defined $\rho_{1} \equiv \rho(1)$. As $z^{\prime}(0, \lambda)$ will serve as our fake shooting function, we require

$$
\begin{aligned}
z^{\prime}(x, \lambda)= & -\frac{\sqrt{\rho(x)}}{\sqrt{\rho}_{1}}\left\{\rho_{1} \sinh \left(\lambda \int_{x}^{1} \rho d s\right)+\cosh \left(\lambda \int_{x}^{1} \rho d s\right)\right\} \\
& -\frac{\rho^{\prime}(x)}{2 \lambda \rho^{3 / 2}(x) \sqrt{\rho}_{1}}\left\{\rho_{1} \cosh \left(\lambda \int_{x}^{1} \rho d s\right)+\sinh \left(\lambda \int_{x}^{1} \rho d s\right)\right\} \\
& +\frac{\sqrt{\rho(x)}(\log \rho)^{\prime}(1)}{2 \lambda \sqrt{\rho}_{1}} \cosh \left(\lambda \int_{x}^{1} \rho d s\right)+\frac{\rho^{\prime}(x)(\log \rho)^{\prime}(1)}{4 \lambda^{2} \rho^{3 / 2}(x) \sqrt{\rho}_{1}} \sinh \left(\lambda \int_{x}^{1} \rho d s\right) \equiv
\end{aligned}
$$




$$
\equiv w_{2}(x, \lambda)+O\left(|\lambda|^{-1}\right) .
$$

We see on inspection that $z(x, \cdot)$ and $z^{\prime}(x, \cdot)$ are asymptotically close to $w_{1}(x, \cdot)$ and $w_{2}(x, \cdot)$. We now show in fact that $\psi(x, \cdot)$ and $\psi^{\prime}(x, \cdot)$ are asymptotically close to $w_{1}(x, \cdot)$ and $w_{2}(x, \cdot)$, where $\psi(x, \lambda)$ is the actual solution to (2.6). By Theorem 5.1 it suffices to work in the band $-\kappa \leq \Re \lambda \leq 0$.

Theorem 6.1. Assume that $\rho \in H^{2}(0,1), \rho_{1} \neq 1$, and $\rho$ satisfies (1.2). Then there exist constants $C_{0}$ and $C_{1}$ such that

$$
\begin{aligned}
\left|\psi(x, \lambda)-w_{1}(x, \lambda)\right| & \leq C_{0}|\lambda|^{-2} \\
\left|\psi^{\prime}(x, \lambda)-w_{2}(x, \lambda)\right| & \leq C_{1}|\lambda|^{-1},
\end{aligned}
$$

uniformly for $0<x<1$ and

$$
-\kappa \leq \Re \lambda \leq 0 \quad|\lambda| \geq \max \left\{1, \frac{\left\|\rho^{\prime}\right\|_{\infty}}{2 \alpha}\right\} .
$$

Proof. We note that $\psi$ satisfies

$$
\psi^{\prime \prime}-q \psi-\lambda^{2} \rho^{2} \psi=-q \psi, \quad \psi(1, \lambda)=1 / \lambda, \psi^{\prime}(1, \lambda)=-1,
$$

and therefore the integral equation

$$
\psi(x, \lambda)=z(x, \lambda)-\int_{x}^{1} K(x, t, \lambda) q(t) \psi(t, \lambda) d t
$$

with $K(x, t, \lambda)=z(x, \lambda) \tilde{z}(t, \lambda)-z(t, \lambda) \tilde{z}(x, \lambda)$, where

$$
\tilde{z}(x, \lambda)=\frac{-1}{\sqrt{\rho_{1} \rho(x)}} \sinh \left(\lambda \int_{x}^{1} \rho d s\right)
$$

satisfies the same differential equation as $z$ but with terminal data $\tilde{z}(1, \lambda)=0$, $\tilde{z}^{\prime}(1, \lambda)=\lambda$. We solve the integral equation (6.6) in series form

$$
\psi(x, \lambda)=\sum_{n=0}^{\infty} S_{n}(x, \lambda),
$$

where $S_{0}=z$ and

$$
\begin{aligned}
& S_{n}(x, \lambda) \\
= & -\int_{x}^{1} K(x, t, \lambda) q(t) S_{n-1}(t, \lambda) d t \\
= & (-1)^{n} \int^{1 \geq t_{1} \geq \cdots \geq t_{n+1}=x} z\left(t_{1}, \lambda\right) \prod_{i=1}^{n}\left[K\left(t_{i+1}, t_{i}, \lambda\right) q\left(t_{i}\right)\right] d t_{1} \cdots d t_{n} .
\end{aligned}
$$


In order to establish the convergence of (6.7) we proceed to derive uniform estimates for the $S_{n}$. To begin, we recall $m=\int_{0}^{1} \rho d x$ and observe that

$$
|z(x, \lambda)| \leq \frac{(\beta+1) e^{\kappa m}}{\alpha|\lambda|}+\frac{\left\|\rho^{\prime}\right\|_{\infty} e^{\kappa m}}{2 \alpha^{2}|\lambda|^{2}} \leq \frac{(\beta+2) e^{\kappa m}}{\alpha|\lambda|}
$$

when $\lambda$ obeys (6.5). Likewise, as

$$
|\tilde{z}(x, \lambda)| \leq \frac{e^{\kappa m}}{\alpha}
$$

it follows that

$$
|K(x, t, \lambda)| \leq \frac{2(\beta+2) e^{2 \kappa m}}{\alpha^{2}|\lambda|}
$$

and hence

$$
\begin{aligned}
\left|S_{n}(x, \lambda)\right| & \leq \frac{(\beta+2) e^{\kappa m}}{\alpha|\lambda|}\left(\frac{2(\beta+2) e^{2 \kappa m}}{\alpha^{2}|\lambda|}\right)^{n} \frac{\|q\|_{1}^{n}}{n !} \\
& \leq \frac{(\beta+2) e^{\kappa m}}{\alpha|\lambda|^{2}} \frac{\left(2 \alpha^{-2}(\beta+2)\|q\|_{2} e^{2 \kappa m}\right)^{n}}{n !} .
\end{aligned}
$$

From the Weierstrass comparison test it now follows that (6.7) converges uniformly for $0 \leq x \leq 1$ and $\lambda$ satisfying (6.5). Moreover,

$$
\begin{aligned}
\mid \psi(x, \lambda) & -w_{1}(x, \lambda)|\leq| \psi(x, \lambda)-z(x, \lambda)|+| z(x, \lambda)-w_{1}(x, \lambda) \mid \\
& \leq\left|\sum_{n=1}^{\infty} S_{n}(x, \lambda)\right|+\frac{e^{\kappa m}\left\|\rho^{\prime}\right\|_{\infty}}{2 \alpha|\lambda|^{2}} \\
& \leq \frac{e^{\kappa m}}{\alpha|\lambda|^{2}}\left(\frac{1}{2}\left\|\rho^{\prime}\right\|_{\infty}+(\beta+2) e^{2 \alpha^{-2}(\beta+2)\|q\|_{2} e^{2 \kappa m}}\right) \\
& \equiv C_{0}|\lambda|^{-2}
\end{aligned}
$$

now establishes (6.3).

Regarding the estimate for $\psi^{\prime}$ we differentiate (6.6) and find

$$
\psi^{\prime}(x, \lambda)-z^{\prime}(x, \lambda)=-\int_{x}^{1} K_{x}(x, t, \lambda) q(t) \psi(t, \lambda) d t,
$$

and so it remains to simply bound $\psi$ and $K_{x}$. With respect to the former

$$
|\psi(x, \lambda)| \leq\left|\psi(x, \lambda)-w_{1}(x, \lambda)\right|+\left|w_{1}(x, \lambda)\right| \leq \frac{C_{0}+(1+\beta) \alpha^{-1} e^{\kappa m}}{|\lambda|},
$$


while the latter requires both

$$
\left|z^{\prime}(x, \lambda)\right| \leq e^{\kappa m}\left(1+\frac{1}{\alpha}+\sqrt{\frac{\beta}{\alpha}}\right)(1+\beta) \quad \text { and } \quad\left|\tilde{z}^{\prime}(x, \lambda)\right| \leq \frac{|\lambda| e^{\kappa m}}{\alpha}(1+\beta)
$$

Assembling these bounds, we find

$$
\begin{aligned}
\left|K_{x}(x, t, \lambda)\right| & \leq\left|z^{\prime}(x, \lambda)\right||\tilde{z}(t, \lambda)|+|z(t, \lambda)|\left|\tilde{z}^{\prime}(x, \lambda)\right| \\
& \leq \frac{e^{2 \kappa m}}{\alpha}\left(1+\frac{1}{\alpha}+\sqrt{\frac{\beta}{\alpha}}\right)(1+\beta)+\frac{e^{2 \kappa m}}{\alpha^{2}}(2+\beta)(1+\beta) \\
& \leq \frac{4 e^{2 \kappa m}}{\alpha^{2}}(1+\beta)^{2} .
\end{aligned}
$$

Now (6.8) yields (6.4),

$$
\begin{aligned}
\mid \psi^{\prime}(x, \lambda) & -w_{2}(x, \lambda)|\leq| \psi^{\prime}(x, \lambda)-z^{\prime}(x, \lambda)|+| z^{\prime}(x, \lambda)-w_{2}(x, \lambda) \mid \\
& \leq \int_{0}^{1}\left|K_{x}(x, t, \lambda)\right||\psi(t, \lambda)||q(t)| d t+\frac{e^{\kappa m}\left\|\rho^{\prime}\right\|_{\infty}}{2|\lambda| \alpha^{2}}(1+\beta)^{2} \\
& \leq \frac{4(1+\beta)^{3} e^{3 \kappa m}}{\alpha^{2}|\lambda|}\left(\left(C_{0}+\alpha^{-1}\right)\|q\|_{2}+\left\|\rho^{\prime}\right\|_{\infty}\right) \\
& \equiv C_{1}|\lambda|^{-1} .
\end{aligned}
$$

On close inspection of the estimate for $S_{n}$ it follows that $d q$ need only be a finite measure, i.e., it suffices to require that $\rho^{\prime}$ simply have finite total variation.

We now show that the zeros of $\psi^{\prime}(0, \lambda)$ are close to the zeros of $w_{2}(0, \lambda)$, these being

$$
\mu_{n}=-\frac{1}{2 m} \log \left|\frac{1+\rho_{1}}{\rho_{1}-1}\right|+\frac{i \pi}{m} \quad\left\{\begin{array}{ll}
n & \text { if } \rho_{1}>1 \\
n+\frac{1}{2} & \text { if } \rho_{1}<1
\end{array} \quad n \in \mathbf{Z}\right.
$$

This is done by choosing $r_{n}$ in

$$
\Gamma_{n}=\left\{\lambda \in \mathbf{C}:\left|\lambda-\mu_{n}\right|=r_{n}\right\}
$$

in such a way that the $\Gamma_{n}$ do not intersect and

$$
\left|\psi^{\prime}(0, \lambda)-w_{2}(0, \lambda)\right|<\left|w_{2}(0, \lambda)\right|, \quad \lambda \in \Gamma_{n}
$$

By the previous theorem it suffices to show that

$$
\left|w_{2}(0, \lambda)\right|>\frac{C_{1}}{|\lambda|}, \quad \lambda \in \Gamma_{n}
$$


We proceed under the assumption that $\rho_{1}>1$, the other case following similarly. If $\lambda \in \Gamma_{n}$ then $\lambda=\mu_{n}+r_{n} e^{i \vartheta}$ where $\vartheta \in[0,2 \pi)$ and

$$
\begin{aligned}
\lambda w_{2}(0, \lambda) & =-\lambda \sqrt{\rho_{0} / \rho_{1}}\left\{\rho_{1} \sinh \left(m\left(\mu_{n}+r_{n} e^{i \vartheta}\right)\right)+\cosh \left(m\left(\mu_{n}+r_{n} e^{i \vartheta}\right)\right)\right\} \\
& =-\lambda \sqrt{\rho_{0} / \rho_{1}}\left(\rho_{1}^{2}-1\right) \sinh \left(m \mu_{n}\right) \sinh \left(m r_{n} e^{i \vartheta}\right) \\
& =(-1)^{n} \sqrt{\rho_{0} / \rho_{1}}\left(\rho_{1}^{2}-1\right) \sinh \left(m \mu_{0}\right)\left(\mu_{n}+r_{n} e^{i \vartheta}\right) \sinh \left(m r_{n} e^{i \vartheta}\right)
\end{aligned}
$$

Hence, if $C^{\prime} \equiv \sqrt{\rho_{0} / \rho_{1}}\left(1-\rho_{1}^{2}\right) \sinh \left(m \mu_{0}\right)$, then

$$
\begin{aligned}
|\lambda|\left|w_{2}(0, \lambda)\right| & >C^{\prime}\left(|n| \pi / m-r_{n}\right)\left|\sinh \left(m r_{n} e^{i \vartheta}\right)\right| \\
& \geq C^{\prime}\left(|n| \pi / m-r_{n}\right)\left(m r_{n}-\frac{1}{2} m^{2} r_{n}^{2}\right) \\
& \geq C^{\prime} r_{n}\left(|n| \pi-m r_{n}\left(1+\frac{1}{2}|n| \pi\right)\right)
\end{aligned}
$$

One makes the obvious guess $r_{n}=\frac{2 C_{1}}{C^{\prime}|n| \pi}$ and finds that

$$
\begin{aligned}
|\lambda|\left|w_{2}(0, \lambda)\right| & \geq C^{\prime} r_{n}\left(|n| \pi-m r_{n}\left(1+\frac{1}{2}|n| \pi\right)\right) \\
& =C_{1}\left(2-\frac{4 C_{1} m}{C^{\prime}|n| \pi}\left(\frac{1}{2}+\frac{1}{|n| \pi}\right)\right) \\
& >C_{1}, \quad \text { when }|n|>N_{1}=\left\lceil\frac{4 C_{1} m}{C^{\prime} \pi}\right\rceil,
\end{aligned}
$$

where $\lceil x\rceil$ denotes the least integer greater than $x$. Furthermore, this choice of $N$ renders $r_{n}<1 /(2 m)$. As the distance between centers of the $\Gamma_{n}$ is $\pi / m$ it follows that the contours are nonintersecting. To capture the remaining eigenvalues we consider

$$
Z_{n}=\left\{\lambda \in \mathbf{C}:\left|\lambda-\mu_{0}\right|=\frac{\pi}{m}\left(n+\frac{1}{2}\right)\right\}
$$

and denote by $N_{2}$ the smallest integer $n>0$ for which $Z_{n}$ encircles the disk of radius $C_{1} / C^{\prime}$ centered at the origin. For each $n$ we note that if $\lambda \in Z_{n}$ then $\left|w_{2}(0, \lambda)\right| \geq C^{\prime}$ while when $n>N_{2}$ we find $|\lambda|>C_{1} / C^{\prime}$ as well. As a result,

$$
|\lambda|\left|w_{2}(0, \lambda)\right|>C_{1}, \quad \lambda \in Z_{n}, n \geq N_{2}
$$

With $N \equiv \max \left\{N_{1}, N_{2}\right\}$, from the Theorem of Rouché follows

Theorem 6.2. If $\rho \in H^{2}(0,1), \rho_{1} \neq 1$, and $\rho$ satisfies (1.2) then $A(\rho)$ has exactly $2 N+1$ eigenvalues, including multiplicity, in $Z_{N}$ and one simple eigenvalue in $\Gamma_{n}$ for $|n|>N$. This exhausts the spectrum of $A$. 
From the estimate $\lambda_{n}=\mu_{n}+O\left(|n|^{-1}\right)$ one easily improves those of Theorem 6.1 at $\lambda=\lambda_{n}$.

Corollary 6.3. If $\rho \in H^{2}(0,1), \rho_{1} \neq 1$, and $\rho$ satisfies (1.2) then, uniformly for $0<x<1$,

$$
\begin{aligned}
\psi\left(x, \lambda_{n}\right) & =w_{1}\left(x, \mu_{n}\right)+O\left(|n|^{-2}\right) \\
\psi^{\prime}\left(x, \lambda_{n}\right) & =w_{2}\left(x, \mu_{n}\right)+O\left(|n|^{-1}\right) .
\end{aligned}
$$

\section{The Root Vectors Comprise a Riesz Basis}

We denote the algebraic multiplicity of $\lambda_{n}$ by $\nu_{n}$ and to $\lambda_{n}$ associate the Jordan Chain of root vectors, $\left\{U_{n, j}\right\}_{j=0}^{\nu_{n}-1}$,

$$
\begin{aligned}
U_{n, 0}(x) & =\psi\left(x, \lambda_{n}\right)\left[1, \lambda_{n}\right], \\
A U_{n, j} & =\lambda_{n} U_{n, j}+U_{n, j-1}, \quad\left\langle U_{n, j}, U_{n, 0}\right\rangle=0, \quad j=1, \ldots, \nu_{n}-1 .
\end{aligned}
$$

Clearly, $U_{n, 0}$ is an eigenvector and the chain is a basis for the root subspace

$$
\mathcal{L}_{n} \equiv\left\{U:\left(A-\lambda_{n}\right)^{\nu_{n}} U=0\right\} .
$$

We construct a biorthogonal sequence to $\left\{\tilde{U}_{n, j}\right\}_{n, j}$ from the eigenvectors of the adjoint, $A^{*}$. We recall that $\sigma(A)=\sigma\left(A^{*}\right)$, including multiplicities, and to $\bar{\lambda}_{n}$ we associate the Jordan Chain of root vectors, $\left\{W_{n, j}\right\}_{j=0}^{\nu_{n}-1}$, where

$$
\begin{aligned}
W_{n, 0}(x) & =\psi\left(x, \bar{\lambda}_{n}\right)\left[1,-\bar{\lambda}_{n}\right], \\
A^{*} W_{n, j} & =\bar{\lambda}_{n} W_{n, j}+W_{n, j-1}, \quad\left\langle W_{n, j}, V_{n, \nu_{n}-1}\right\rangle=0, \quad j=1, \ldots, \nu_{n}-1 .
\end{aligned}
$$

Observe that $W_{n, 0}$ is an eigenvector for $A^{*}$ and that the subsequent $W_{n, j}$ are uniquely determined so long as $\left\langle W_{n, 0}, V_{n, \nu_{n}-1}\right\rangle \neq 0$. In addition, the chain $\left\{W_{n, j}\right\}_{j=0}^{\nu_{n}-1}$ is a basis for the root subspace

$$
\mathcal{L}_{n}^{*} \equiv\left\{W:\left(A^{*}-\bar{\lambda}_{n}\right)^{\nu_{n}} W=0\right\} .
$$

Lemma 7.1. If $\rho \in H^{2}(0,1), \rho_{1} \neq 1$, and $\rho$ satisfies (1.2) then there exists $a c>0$ such that

$$
\left|\left\langle U_{n, p}, W_{j, k}\right\rangle\right|=\left|\left\langle U_{n, p}, W_{n, \nu_{n}-1-p}\right\rangle\right| \delta_{n, j} \delta_{\nu_{n}-1-p, k} \geq c \delta_{n, j} \delta_{\nu_{n}-1-p, k} .
$$

Proof. The biorthogonality is an algebraic result that follows essentially by construction. For details see [2, Lemma 6.2]. The fact that $c>0$, i.e., that the 
two sequences may be binormalized, is a consequence of the asymptotic formulas of Corollary 6.3. In particular, we recall from Theorem 6.2 that $\mu_{n}=1$ for $n>|N|$ and proceed for such $n$ to establish

$$
\begin{aligned}
\left\langle U_{n, 0}, W_{n, 0}\right\rangle= & \left\langle\psi\left(x, \lambda_{n}\right)\left[1, \lambda_{n}\right], \psi\left(x, \bar{\lambda}_{n}\right)\left[1,-\bar{\lambda}_{n}\right]\right\rangle \\
= & \int_{0}^{1}\left(\psi^{\prime}\left(x, \lambda_{n}\right)\right)^{2}-\lambda_{n}^{2} \rho^{2} \psi^{2}\left(x, \lambda_{n}\right) d x \\
= & \int_{0}^{1} w_{2}^{2}\left(x, \mu_{n}\right)-\mu_{n}^{2} \rho^{2} w_{1}^{2}\left(x, \mu_{n}\right) d x+O\left(|n|^{-1}\right) \\
= & \int_{0}^{1} \frac{\rho}{\rho_{1}}\left(\left\{\rho_{1} \sinh \left(\mu_{n} \int_{x}^{1} \rho d s\right)+\cosh \left(\mu_{n} \int_{x}^{1} \rho d s\right)\right\}^{2}\right. \\
& \left.-\left\{\rho_{1} \cosh \left(\mu_{n} \int_{x}^{1} \rho d s\right)+\sinh \left(\mu_{n} \int_{x}^{1} \rho d s\right)\right\}^{2}\right) d x+O\left(|n|^{-1}\right) \\
= & \frac{m}{\rho_{1}}\left(1-\rho_{1}^{2}\right)+O\left(|n|^{-1}\right)
\end{aligned}
$$

As $\rho_{1} \neq 1$ it follows that $\left|\left\langle U_{n, 0}, W_{n, 0}\right\rangle\right| \geq \frac{m}{2 \rho_{1}}\left(1-\rho_{1}^{2}\right)$ for $n$ of sufficient magnitude.

We may therefore binormalize,

$$
\tilde{U}_{n, 0}(x)=\left\langle U_{n, 0}, W_{n, 0}\right\rangle^{-1 / 2} U_{n, 0}(x)=U_{n, 0}(x)+O(1 /|n|),
$$

and

$$
\tilde{W}_{n, 0}(x)=\left\langle U_{n, 0}, W_{n, 0}\right\rangle^{-1 / 2} W_{n, 0}(x)=W_{n, 0}(x)+O(1 /|n|)
$$

for $|n|>N$. Having demonstrated completeness, in order to invoke Bari's Theorem it suffices to check that $\left\{\left\langle\tilde{U}_{n, 0},[f, g]\right\rangle\right\}_{n} \in \ell^{2}(\mathbf{C})$ for each $[f, g] \in V$. Drawing once again on Corollary 6.3 we find

$$
\begin{aligned}
\left\langle\tilde{U}_{n, 0},[f, g]\right\rangle= & \int_{0}^{1} \psi^{\prime}\left(x, \lambda_{n}\right) \bar{f}^{\prime}+\rho^{2} \lambda_{n} \psi\left(x, \lambda_{n}\right) \bar{g} d x+O\left(|n|^{-1}\right) \\
= & \int_{0}^{1} w_{2}\left(x, \mu_{n}\right) \bar{f}^{\prime}+\rho^{2} \mu_{n} w_{1}\left(x, \mu_{n}\right) \bar{g} d x+O\left(|n|^{-1}\right) \\
= & \int_{0}^{1} \frac{\sqrt{\rho}}{\sqrt{\rho_{1}}}\left(\sinh \left(\mu_{n} \int_{x}^{1} \rho d s\right)\left(\rho \bar{g}-\rho_{1} \bar{f}^{\prime}\right)\right. \\
& \left.\quad+\cosh \left(\mu_{n} \int_{x}^{1} \rho d s\right)\left(\rho_{1} \rho \bar{g}-\bar{f}^{\prime}\right)\right) d x+O\left(|n|^{-1}\right) .
\end{aligned}
$$


Its square summability is evidently determined by that of terms of the form

$$
\int_{0}^{1} h(x) \sin (n \pi \xi(x)) d x, \text { where } \quad \xi(x)=\frac{\int_{x}^{1} \rho d s}{\int_{0}^{1} \rho d s}
$$

for $h \in L^{2}(0,1)$. On performing the change of variables $t=\xi(x)$ we find

$$
\int_{0}^{1} h(x) \sin (n \pi \xi(x)) d x=\int_{0}^{1} \frac{h\left(\xi^{-1}(t)\right)}{\rho\left(\xi^{-1}(t)\right)} \sin (n \pi t) d t,
$$

without doubt an element of $\ell^{2}(\mathbf{C})$. Hence, the following result:

Theorem 7.2. If $\rho \in H^{2}(0,1), \rho_{1} \neq 1$, and $\rho$ satisfies (1.2) then the root vectors of $A(\rho)$ comprise a Riesz basis for $V$ and $\omega(\rho)=\mu(\rho)$.

The proof of the fact that the decay rate indeed coincides with the spectral abscissa is exactly as given in [2, Theorem 2.1].

\section{Optimal Design}

With the previous theorem, the minimization of $\omega(\rho)$ over

$$
a d=\left\{\rho: 0<\alpha \leq \rho(x),\left|1-\rho_{1}\right| \geq \gamma>0,\|\rho\|_{H^{2}} \leq C\right\}
$$

takes on the flavor of a pole placement problem.

Theorem 8.1. The decay rate, $\rho \mapsto \omega(\rho)$, achieves its minimum over ad.

Proof. In view of Theorem 5.1 we know that $\omega(\rho)$ has a finite infimum over $a d$. Let us denote it by $\check{\omega}$ and choose a minimizing sequence $\left\{\rho_{n}\right\}_{n} \in a d$. As this sequence is uniformly bounded in $H^{2}(0,1)$ it follows that there exists a $\check{\rho} \in$ ad and a subsequence (that we neglect to relable) for which $\rho_{n} \rightarrow \check{\rho}$ strongly in $L^{\infty}(0,1)$

We now show that $\omega(\check{\rho})=\check{\omega}$ by considering

$$
\omega(\check{\rho}) \geq \frac{-1}{2 \int_{0}^{1} \check{\rho}} \log \left|\frac{1+\check{\rho}_{1}}{\check{\rho}_{1}-1}\right| .
$$

If in fact equality holds we simply note that

$$
\omega\left(\rho_{n}\right) \geq \frac{-1}{2 \int_{0}^{1} \rho_{n}} \log \left|\frac{1+\rho_{n}(1)}{\rho_{n}(1)-1}\right| \rightarrow \frac{-1}{2 \int_{0}^{1} \check{\rho}} \log \left|\frac{1+\check{\rho}_{1}}{\check{\rho}_{1}-1}\right|=\omega(\check{\rho}) .
$$


Hence, in this case $\check{\omega} \geq \omega(\check{\rho})$ and $\check{\rho}$ is a minimizer.

If instead inequality prevails in (8.1) we turn to the half-plane

$$
H \equiv\left\{\lambda: \omega(\check{\rho})-\frac{1}{2 \int_{0}^{1} \check{\rho}} \log \left|\frac{1+\check{\rho}_{1}}{\check{\rho}_{1}-1}\right| \leq 2 \Re \lambda\right\},
$$

and note the existence of finite $n_{1}$ and $n_{2}$ such that for $|n|>n_{1}$ one finds exactly $n_{2}$ points in $\sigma\left(A\left(\rho_{n}\right)\right) \cap H$. That $\omega\left(\rho_{n}\right) \rightarrow \omega(\check{\rho})$ now follows from the continuity of a finite system of eigenvalues, see Kato [8, IV.3.5], under the uniform convergence of $A\left(\rho_{n}\right)^{-1}$ to $A(\check{\rho})^{-1}$ in $L(X)$. To see the latter, note that

$$
\begin{gathered}
\left\|A\left(\rho_{n}\right)^{-1}-A(\check{\rho})^{-1}\right\|_{L(X)}=\sup _{\left\|\left[f_{1}, f_{2}\right]\right\|_{X}=1}\left\|A\left(\rho_{n}\right)^{-1}\left[f_{1}, f_{2}\right]-A(\check{\rho})^{-1}\left[f_{1}, f_{2}\right]\right\|_{X} \\
=\sup _{\left\|\left[f_{1}, f_{2}\right]\right\|_{X}=1} \| \int_{0}^{x}\left(\rho_{n}^{2}(s)-\check{\rho}^{2}(s)\right) f_{2}(s) d s- \\
\quad \int_{0}^{1}\left(\rho_{n}^{2}(s)-\check{\rho}^{2}(s)\right)\left((1-s) f_{2}(s)+f_{1}(s)\right) d s \|_{H^{1}} \\
\leq 4\left\|\rho_{n}^{2}-\check{\rho}^{2}\right\|_{\infty}
\end{gathered}
$$

for all $\left[f_{1}, f_{2}\right] \in X$. The result now follows from the $L^{\infty}(0,1)$ convergence of $\rho_{n}$ to $\check{\rho}$.

\section{Comments}

We have remarked that Majda established conditions under which solutions may not vanish as well as rough bounds on the associated spectra for (2.12), the $n$-dimensional wave equation with both internal and boundary dissipation. Majda in [15] also shows that existence of vanishing solutions implies noncompleteness of the associated collection of root vectors.

The eigenvalues for such problems in particular planar sets have been estimated by Quinn and Russell [18] and Zhou and Chen [23] under the assumption of zero internal damping and piecewise constant boundary damping. In particular, Quinn and Russell [18] address

$$
\begin{gathered}
u_{t t}-\Delta u=0, \quad u\left(x_{1}, 0, t\right)=u\left(0, x_{2}, t\right)=0 \\
u_{x_{1}}\left(\pi, x_{2}, t\right)+\gamma_{1} u_{t}\left(\pi, x_{2}, t\right)=0 \\
u_{x_{2}}\left(x_{1}, \pi, t\right)+\gamma_{2} u_{t}\left(x_{1}, \pi, t\right)=0
\end{gathered}
$$


on the square $0<x_{1}, x_{2}<\pi$. In [23] like calculations are carried out on the rectangle and disk. Neither [18] nor [23] pursue the completeness of the associated root vectors.

Regarding technique, one finds little intersection between the extensive literature on the decay of solutions of the $n$-dimensional wave equation with boundary damping (see, e.g., Komornik and Zuazua [9] and Lebeau [13]) and the spectral approach we have taken here and in [2]. Roughly, what our approach gives up in terms of generality it makes up in terms of precision.

\section{Appendix}

In this section, for the sake of completeness, we give a simple proof of the exponential decay of solutions of the dissipative wave equation (1.1) when $\rho$ is uniformly positive and of bounded variation.

Theorem 10.1. If $\rho \geq \alpha>0$ and $\rho$ has finite total variation then there exist positive constants $C>0$ and $\omega>0$ such that

$$
E(t) \leq C E(0) e^{-2 \omega t}
$$

holds for every solution of (1.1), where the energy $E$ is given by

$$
E(t)=\frac{1}{2} \int_{0}^{1} u_{x}^{2}(x, t)+\rho^{2}(x) u_{t}^{2}(x, t) .
$$

Proof. First of all we observe that it is sufficient to prove the existence of some time $\tau>0$ and some constant $C_{0}>0$ such that

$$
E(\tau) \leq C_{0} \int_{0}^{\tau} u_{t}^{2}(1, t) d t
$$

for all solutions of (1.1). Indeed, multiplying (1.1) by $u_{t}$ and integrating in $(0,1) \times(0, \tau)$ we find

$$
E(0)-E(\tau)=\int_{0}^{\tau} u_{t}^{2}(1, t) d t
$$

Combining (10.2) and (10.3) we deduce that

$$
E(\tau) \leq \frac{C_{0}}{C_{0}+1} E(0) .
$$

In view of (10.4) and as a consequence of the semigroup property we deduce that (10.1) holds with

$$
C=\frac{C_{0}+1}{C_{0}} \quad \text { and } \quad \omega=\frac{\log \left(\left(C_{0}+1\right) / C_{0}\right)}{2 \tau} .
$$


In order to prove (10.2) we define the "sideways" energy

$$
F(x)=\frac{1}{2} \int_{\beta-\beta x}^{\tau-\beta+\beta x} u_{x}^{2}(x, t)+\rho^{2}(x) u_{t}^{2}(x, t) d t
$$

with $\beta=\|\rho\|_{\infty}$ and for some $\tau>2 \beta$. We have

$$
\begin{aligned}
F^{\prime}(x)= & \int_{\beta-\beta x}^{\tau-\beta+\beta x}\left\{u_{x x}(x, t) u_{x}(x, t)+\rho^{2}(x) u_{x t}(x, t) u_{t}(x, t)\right. \\
& \left.+\rho(x) \rho^{\prime}(x) u_{t}^{2}(x, t)\right\} d t-\frac{\beta}{2}\left\{u_{x}^{2}(x, \beta-\beta x)+\rho^{2}(x) u_{t}^{2}(x, \beta-\beta x)\right. \\
& \left.+u_{x}^{2}(x, \tau-\beta+\beta x)+\rho^{2}(x) u_{t}^{2}(x, \tau-\beta+\beta x)\right\} .
\end{aligned}
$$

Integrating by parts we get

$$
\begin{aligned}
F^{\prime}(x)= & \int_{\beta-\beta x}^{\tau-\beta+\beta x} \rho(x) \rho^{\prime}(x) u_{t}^{2}(x, t) d t+\left.\rho^{2}(x) u_{t}(x, t) u_{x}(x, t)\right|_{t=\beta-\beta x} ^{t=\tau+\beta-\beta x} \\
& -\frac{\beta}{2}\left\{u_{x}^{2}(x, \beta-\beta x)+\rho^{2}(x) u_{t}^{2}(x, \beta-\beta x)+u_{x}^{2}(x, \tau-\beta+\beta x)\right. \\
& \left.+\rho^{2}(x) u_{t}^{2}(x, \tau-\beta+\beta x)\right\} .
\end{aligned}
$$

Hence, by our choice of $\beta$,

$$
\left|F^{\prime}(x)\right| \leq \int_{\beta-\beta x}^{\tau-\beta+\beta x} \rho(x)\left|\rho^{\prime}(x)\right| u_{t}^{2}(x, t) d t \leq 2 \alpha^{-1}\left|\rho^{\prime}(x)\right| F(x) .
$$

With Gronwall's inequality we now deduce that

$$
F(x) \leq e^{(2 / \alpha) \int_{x}^{1}\left|\rho^{\prime}\right| d s} F(1) \leq e^{(2 / \alpha)\left\|\rho^{\prime}\right\|_{1}} F(1)
$$

for each $x \in(0,1)$. Thus

$$
\begin{aligned}
(\tau-2 \beta) E(\tau) & \leq(\tau-2 \beta) E(\tau-2 \beta) \\
& \leq \frac{1}{2} \int_{0}^{1} \int_{\beta}^{\tau-\beta} u_{x}^{2}(x, t)+\rho^{2}(x) u_{t}^{2}(x, t) d t d x \\
& \leq \int_{0}^{1} F(x) \\
& \leq e^{(2 / \alpha)\left\|\rho^{\prime}\right\|_{1}} F(1) \\
& =\frac{1}{2} e^{(2 / \alpha)\left\|\rho^{\prime}\right\|_{1}} \int_{0}^{\tau} \rho^{2}(1) u_{t}^{2}(1, t)+u_{x}^{2}(1, t) d t \\
& =\left(\rho^{2}(1)+1\right) e^{(2 / \alpha)\left\|\rho^{\prime}\right\|_{1}} \int_{0}^{\tau} u_{t}^{2}(1, t) d t .
\end{aligned}
$$


Therefore (10.2) holds with

$$
C_{0}=\frac{\rho^{2}(1)+1}{2(\tau-2 \beta)} e^{(2 / \alpha)\left\|\rho^{\prime}\right\|_{1}} .
$$

The argument above applies to $\rho$ smooth enough (for instance $\rho \in C^{1}(0,1)$ ). However, in view of the structure of the constant $C_{0}$ in (10.5), by density, the result holds for any $\rho$ satisfying the stated hypotheses.

\section{Acknowledgements}

We thank Natalia Alexandrov for an English translation of [12]. This work was done while the second author was visiting the Department of Computational and Applied Mathematics of Rice University (Houston) with the support of Ministerio de Educación y Ciencia (Spain). The first author has been supported by NSF Grant DMS-9258312. The second author was also partially supported by grants PB90-0245 and PB93-1203 of the DGIYT (Spain) and SC1*-0732 of the ECC.

\section{REFERENCES}

[1] M. Cherkaoui, Estimation optimale du taux de décroissance de l'énergie pour une équation des ondes avec contrôle frontière, (Preprint).

[2] S. Cox And E. Zuazua, The rate at which energy decays in a damped string, Commun. in PDE 19(1\&2) (1994), 213-243.

[3] H. Dym and H. P. McKean, Gaussian Processes, Function Theory, and the Inverse Spectral Problem, Academic Press, New York.

[4] I. C. Goberg And M. G. Krein, Introduction to the Theory of Linear Nonselfadjoint Operators, Amer. Math. Soc., Providence, 1969.

[5] S. V. HRušČEv, The Regge problem for strings, unconditionally convergent eigenfunction expansions, and unconditional bases of exponentials in $L^{2}(-T, T)$, Operator theory 14 (1985), 67-85.

[6] S. V. Hruščev, N. K. Nikol'skit and B. S. Pavlov, Unconditional bases of exponentials and on reproducing kernels, Complex Analysis and Spectral Theory, LNM \# 864 (V. P. Havin and N. K. Nikol'skii, eds.), Springer, New York, 1981.

[7] I. C. Kac And M. G. Krein, On the Spectral Function of the String, Amer. Math. Soc. Translations, Ser. 2, Vol. 103, 1974, pp. 19-102.

[8] T. Kato, Perturbation Theory for Linear Operators, 2nd ed., Springer-Verlag, New York, 1984.

[9] V. Komornik and E. Zuazua, A Direct method for the boundary stabilization of the wave equation, J. Math. Pures et Appl. 69(1) (1990), 33-55.

[10] M. G. KRein AND H. LANGeR, On some mathematical principles in the linear theory of damped oscillations of continua I, Integral Equations and Operator Theory 1/3 (1978), 364-399.

[11] M. G. Krein And A. A. Nudel'man, On direct and inverse problems for the boundary dissipation frequencies of a nonuniform string, Soviet Math. Dokl. 20 (4) (1979), 838-841. 
[12] M. G. Krein And A. A. Nudel'man, Some spectral properties of a nonhomogeneous string with a dissipative boundary condition, (in Russian), J. Operator Theory 22 (1989), 369-395.

[13] G. Lebeau, Contrôle et stabilisation hyperboliques, Séminaire EDP, Ecole Polytechnique (1989-1990), XVI-1-XVI-14.

[14] B. Ja. Levin, Distribution of Zeros of Entire Functions, Amer. Math. Soc., Providence, 1964.

[15] A. MAJDA, Disappearing solutions for the dissipative wave equation, Indiana Univ. Math. J. 24 (1975), 1119-1133.

[16] A. MAJDA, The location of the spectrum for the dissipative acoustic operator, Indiana Univ. Math. J. 25 (1976), 973-987.

[17] N. K. Nikol'skit, Treatise on the Shift Operator, Springer, New York, 1985.

[18] J. P. Quinn AND D. L. Russell, Asymptotic stability and energy decay rates for solutions of hyperbolic equations with boundary damping, Proc. Roy. Soc. Edinburgh, Sect. A 77 (1977), 97-127.

[19] J. RAUCh AND M. TAYlor, Exponential decay of solutions to hyperbolic equations in bounded domains, Indiana J. Math. 24 (1974), 79-83.

[20] P. RideAu, Contrôle d'un assemblage de poutres flexibles par des capteurs actionneurs ponctuels:étude du spectre du système, Thèse, Ecole Nat. Sup. des Mines de Paris, SophiaAntipolis, France, 1985.

[21] W. W. Symes, On the relation between coefficient and boundary values for solutions of Webster's horn equation, SIAM J. Math. Anal. 17(6) (1986), 1400-1420.

[22] M. A. Shubov, Asymptotics of resonances and geometry of resonance states in the problem of scattering of acoustic waves by a spherically symmetric inhomogeneity of the density, Differential and Integral Eqns. (to appear).

[23] J. Zhou And G. Chen, The wave method for determining the asymptotic damping rates of eigenmodes I: The wave equation on a rectangular or circular domain, SIAM J. Cont. Optim. 29(3) (1991), 656-677.

[24] E. ZuAzuA, Exact controllability for the semilinear wave equation in one space dimension, Ann. IHP. Analyse Non Linéaire 10 (1993), 109-129.

Steven J. Cox

Department of Computational and Applied Math.

Rice University

P.O. Box 1892

Houston, TX 77251, U. S. A.
ENRIQUe ZuAZuA

Departamento de Matemática Aplicada Universidad Complutense de Madrid 28040, Madrid SPAIN

Received: January 9th, 1995. 